FINAL SCIENTIFIC TECHNICAL REPORT

\title{
DENSE MULTIPHASE FLOW SIMULATION: CONTINUUM MODEL FOR POLY-DISPERSED SYSTEMS USING KINETIC THEORY
}

FEDERAL CONTRACT NUMBER: DE-NT0001740

Principal Investigator Moses N. Bogere, Ph.D.

UNIVERSITY OF PUERTO RICO - MAYAGUEZ

Date of report: November 30, 2011

Prepared for:

U.S. Department of Energy National Energy Technology Laboratory 


\section{DISCLAIMER}

“This report was prepared as an account of work sponsored by an agency of the United States Government. Neither the United States Government nor any agency thereof, nor any of their employees, makes warranty, express or implied, or assumes any legal liability or responsibility for the accuracy, completeness, or usefulness of any information, apparatus, product, or process disclosed, or represents that its use would not infringe privately owned rights. Reference herein to any specific commercial product, process, or service by trade name, trademark, manufacturer, or otherwise does not necessarily constitute or imply its endorsement, recommendation, or favoring by the United States Government or any agency thereof. The views and opinions of authors expressed herein do not necessarily state or reflect those of the United States Government or any agency thereof." 


\section{EXECUTIVE SUMMARY}

The overall objective of the project was to verify the applicability of the FCMOM approach to the kinetic equations describing the particle flow dynamics. For monodispersed systems the fundamental equation governing the particle flow dynamics is the Boltzmann equation.

During the project, the FCMOM was successfully applied to several homogeneous and in-homogeneous problems in different flow regimes, demonstrating that the FCMOM has the potential to be used to solve efficiently the Boltzmann equation. However, some relevant issues still need to be resolved, i.e. the homogeneous cooling problem (inelastic particles cases) and the transition between different regimes.

In this report, the results obtained in homogeneous conditions are discussed first. Then a discussion of the validation results for in-homogeneous conditions is provided. And finally, a discussion will be provided about the transition between different regimes.

Alongside the work on development of FCMOM approach studies were undertaken in order to provide insights into anisotropy or particles kinetics in riser hydrodynamics. This report includes results of studies of multiphase flow with unequal granular temperatures and analysis of momentum re-distribution in risers due to particle-particle and fluid-particle interactions. The study of multiphase flow with unequal granular temperatures entailed both simulation and experimental studies of two particles sizes in a riser and, a brief discussion of what was accomplished will be provided. And finally, a discussion of the analysis done on momentum re-distribution of gas-particles flow in risers will be provided. In particular a discussion of the remaining work needed in order to improve accuracy and predictability of riser hydrodynamics based on two-fluid models and how they can be used to model segregation in risers. 


\section{INTRODUCTION}

Coal-based power plants continue to play a critical role in the national sustainable energy program. Significant improvements in our understanding of the chemical and physical processes involved in the conversion and utilization of coal are necessary to achieve the national goal of developing cost-effective, environmentally acceptable, efficient processes for producing electricity and transportation fuels through the use of coal. Computer simulation of coal conversion processes not only provides an excellent tool for scale up, but also significantly improves the understanding and ability to predict the performance of such processes. The simulation and design of a CFB reactor (riser) requires one to account for gas-particles flows in which a system of particles whose sizes are broadly distributed. Up to date, two main approaches have been proposed to model the fluid dynamics of a poly-dispersed system of particles (PSP): 1) the multi-size approach; 2) the continuous particle size distribution (PSD) approach. The major shortcoming of using a multi-size approach is the requirement of significant computational time when several particulate phases are considered. In this project, the PSD approach was taken in order to minimize that computational effort. A model based on the continuous PSD approach was used to describe the fluid dynamics of a PSP. Then the FCMOM numerical technique was validated for solving the model for a number of cases. At the same time, continued examination of anisotropy or particle kinetics in risers was undertaken through simulation (of multiphase flow with unequal granular temperatures) and through analysis of momentum re-distribution in risers (and causes for segregation). The combined efforts were targeted to bring us closer to the ultimate objective of the original proposed work: "to derive the governing and constitutive equations based on the kinetic theory for PSP by using a generalized Boltzmann equation (GBE) in which both particle size and velocity distributions is taken into account”.

\section{RESULTS AND DISCUSSION OF ACCOMPLISHED WORK}

This report includes a narrative of the methodology used to develop the FCMOM numerical technique and a discussion of some results of validation cases. This will then be followed with discussion of work undertaken on studies of anisotropy or particles kinetics in risers. 


\section{FCMOM validation for the Boltzmann equation in homogeneous conditions}

The validation of the FCMOM is developed in a 2-D domain. The bi-dimensional Boltzmann equation in homogeneous conditions is the following equation [1]:

$$
\frac{\partial f\left(\mathbf{c}_{\mathbf{1}}, t\right)}{\partial t}=\int_{\mathbf{g} \bullet \mathbf{k}>0} \int\left[\frac{1}{e^{2}} \cdot f\left(\mathbf{c}_{1}^{\prime \prime}, t\right) \cdot f\left(\mathbf{c}_{2}^{\prime \prime}, t\right)-f\left(\mathbf{c}_{1}, t\right) \cdot f\left(\mathbf{c}_{2}, t\right)\right] \cdot D \cdot(\mathbf{g} \cdot \mathbf{k}) \cdot d \mathbf{k} \cdot d \mathbf{c}_{\mathbf{2}}
$$

In Eq. (1), $t$ is the time, $\mathbf{c}_{\mathbf{1}}$ and $\mathbf{c}_{\mathbf{2}}$ are the velocities of the colliding particles, $f$ is the particle velocity distribution function, $D$ is the particle diameter, $e$ is the restitution coefficient, $\mathbf{g}$ denotes the relative velocity between the two colliding particles, $\mathbf{k}$ is the unit vector directed from the center of the first particle to the center of the second particle, and $\mathbf{c}_{\mathbf{1}}$, $\mathbf{c}_{\mathbf{2}}$, denote the particle velocities after the collision.

The objective was to solve Eq. (1) using the FCMOM numerical technique. The reason for this choice is that, on one hand, the FCMOM is an efficient technique to solve accurately population balance equations [2,3] and recently the technique was used to solve bivariate population balance equations [3]. On the other hand, Eq. (1) is a bivariate population balance equation [4] whose internal variables are the two components of the particle velocity.

In the FCMOM approach, a finite domain of the internal coordinates (particle velocities) is initially defined. For simplicity square domains was considered, in which the maximum velocity is a constant $c^{\max }$. After a coordinate transformation, the velocity space is transformed into the dimensionless velocity space, which is the square $[-1,1 ;-1,1]$. On the hand, $\overline{c_{1, x}} \& \overline{c_{1, y}}$ are the dimensionless components of the first particle velocity, as previously reported [3]. The dimensionless moments $\mu_{i_{x}, i_{y}}$ are defined as follows:

$$
\mu_{i_{x}, i_{y}}=\int_{-1}^{1} \int_{-1}^{1} \overline{f^{\prime}}\left(\overline{c_{1, x}}, \overline{c_{2, y}}, t\right) \cdot\left(\overline{c_{1, x}}\right)^{i_{x}} \cdot\left(\overline{c_{1, y}}\right)^{i_{y}} \cdot d \overline{c_{1, x}} \cdot d \overline{c_{1, y}}
$$

In Eq. (2), $\overline{f^{\prime}}$ is the dimensionless particle velocity distribution ( $f$ divided by a scale factor $f_{s c}$, as reported in [3]).

From the Eq. (1), the evolution equations of the dimensionless moments are derived as shown below.

$$
\begin{aligned}
& \frac{\partial \mu_{i_{x}, i_{y}}}{\partial t}= \\
& =D \cdot f_{s c} \cdot\left(c^{\max }\right)^{2} \cdot \int\left[\frac{1}{e^{2}} \cdot \overline{f^{\prime}\left(\overline{\mathbf{c}_{1}^{\prime \prime}}, t\right)} \cdot \overline{f^{\prime}\left(\overline{\mathbf{c}_{2}^{\prime \prime}}, t\right)}-\overline{f^{\prime}\left(\overline{\mathbf{c}_{1}}, t\right)} \cdot \overline{f^{\prime}\left(\overline{\mathbf{c}_{2}}, t\right)}\right] \cdot\left(\overline{c_{1, x}}\right)^{i_{x}} \cdot\left(\overline{c_{1, y}}\right)^{i_{y}} \cdot(\mathbf{g} \cdot \mathbf{k}) \cdot d \mathbf{k} \cdot d \overline{\mathbf{c}_{1}} \cdot d \overline{\mathbf{c}_{2}}
\end{aligned}
$$


The integrals on the right-hand side are multiple (5 variables) integrals and are subject to the condition $(\mathbf{g} \cdot \mathbf{k})>0$.

The evolution equation of the dimensionless moments can be easily solved after the dimensionless particle velocity distribution is expressed as a function of the dimensionless particle velocities using Legendre polynomials ([3]) and that the integrals on the right-hand side of Eq. (3) are solved numerically.

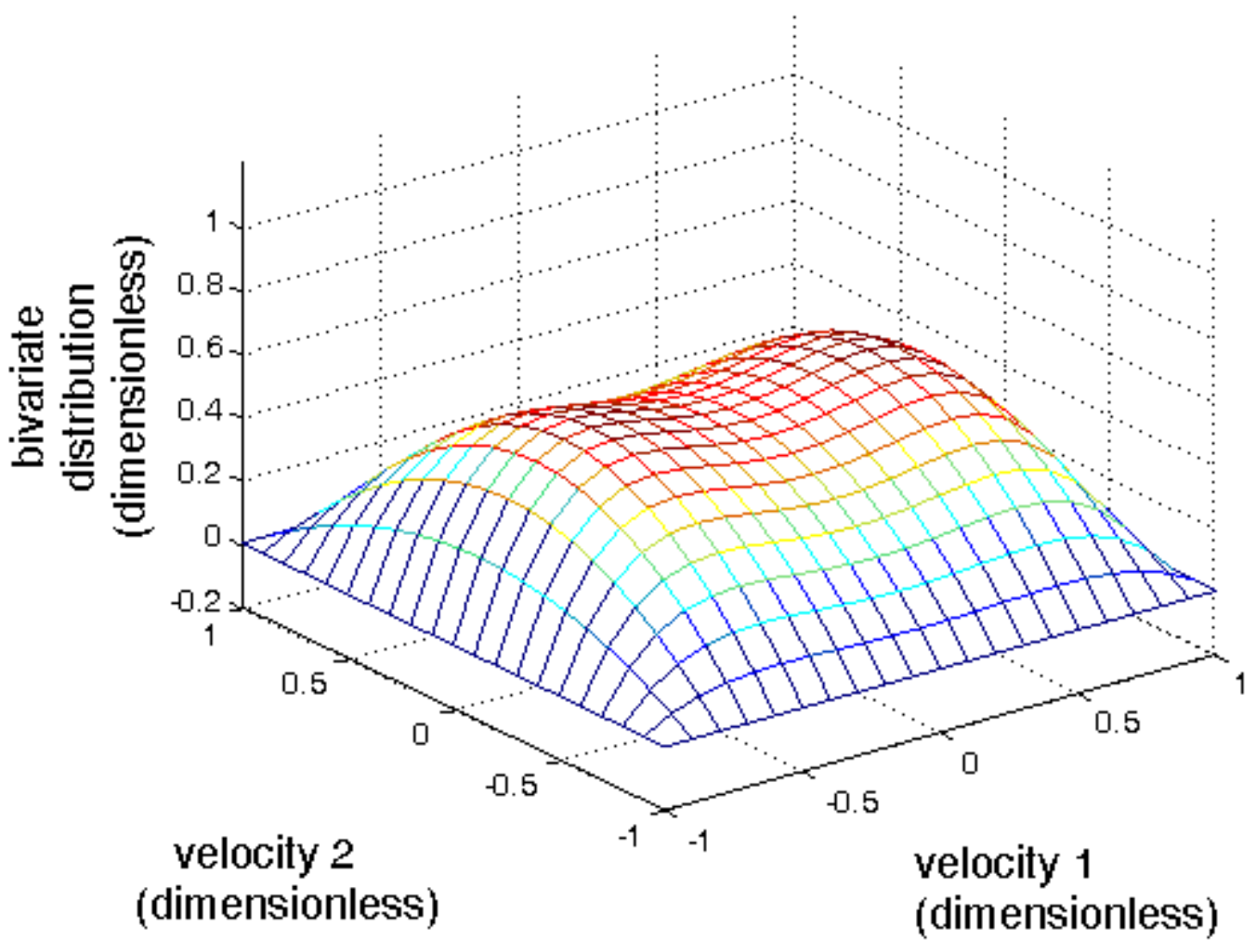

Figure 1 


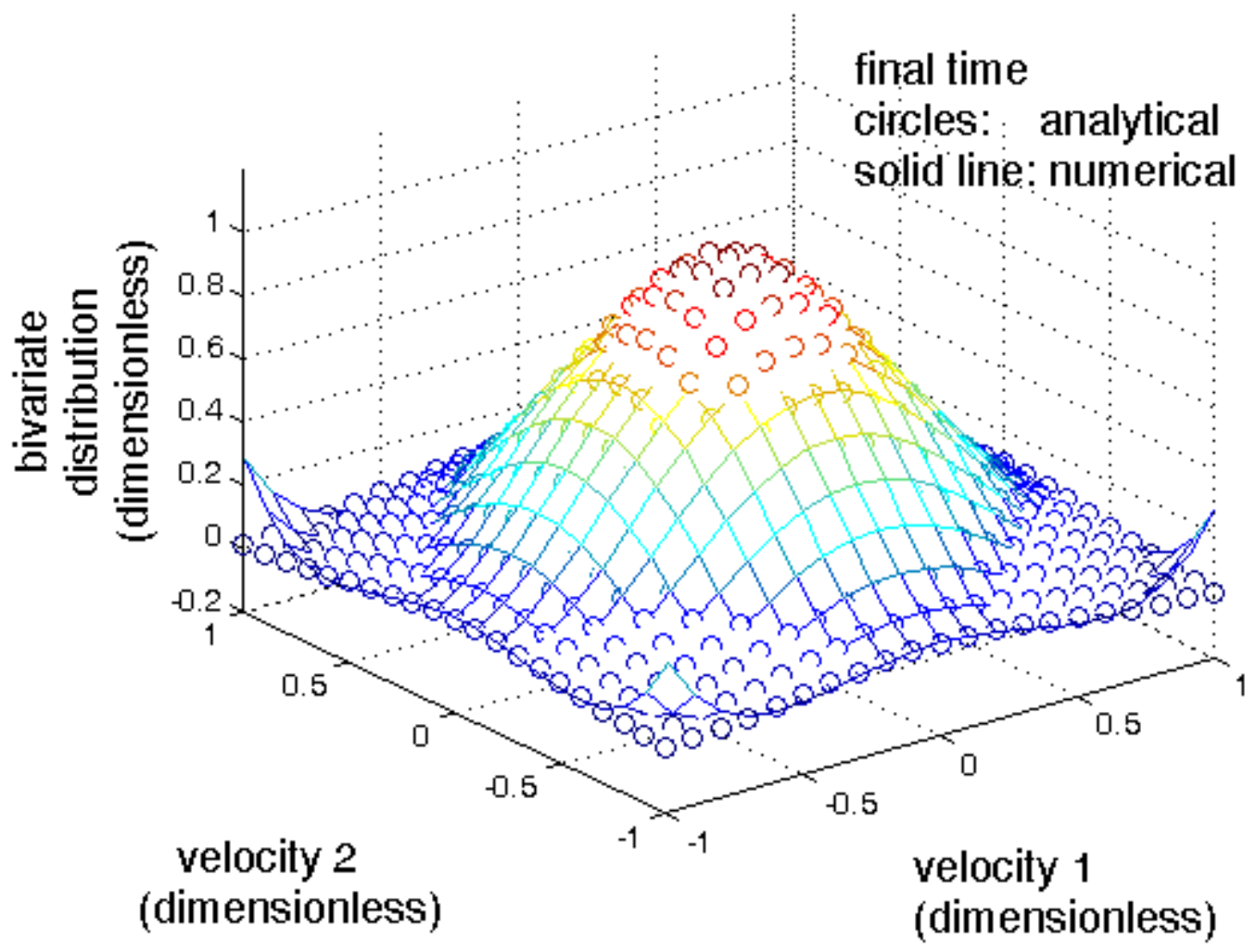

Figure 2

Some results for the elastic case $(e=1)$ are shown in Figures 1-2. In this case, starting from a non-equilibrium particle velocity distribution, it is expected that the system relaxes asymptotically to the Maxwellian distribution function. The integrals on the right-hand side of Eq. (3) were computed and Eq. (3) was solved by using moments up to fifth order. The particle diameter used in the simulations was 100 microns, and the granular temperature was approximately $0.2 \mathrm{~m}^{2} / \mathrm{s}^{2}$.

In Figure 1, the initial dimensionless particle velocity distribution is shown. It is clearly a nonMaxwellian distribution. Figure 2 illustrates a comparison between the numerical results obtained by the FCMOM technique and the exact Maxwellian distribution. The numerical solution approaches the final exact solution however the particle velocity distribution reconstruction is not accurate, due to the presence of oscillations. Such oscillations are present because the numerical solution was obtained for moments up to the fifth order. It is a typical behavior of the FCMOM technique to show oscillations in the numerical solution for relatively low order of moments [2]; typically such oscillations disappear by increasing the order of the moments to 8-10 (depending on the problem considered).

A second set of simulations were conducted by using moments up to the eighth order. In the simulations, starting from a non-equilibrium particle velocity distribution function, it is expected that the system relaxes asymptotically to the Maxwellian distribution function. The particle 
diameter used in the simulations was 100 microns, and the granular temperature was approximately $0.144 \mathrm{~m}^{2} / \mathrm{s}^{2}$. The results of these studies are illustrated in Figures 3-6.

The results of the initial dimensionless particle-velocity distribution function are illustrated in Figure 3. It is clearly a non-Maxwellian distribution. A comparison of the numerical results (final dimensionless particle velocity distribution function) obtained by the FCMOM to the Maxwellian distribution was also made and the results of which is shown in Figures 4-6. The results in Figure 4 are for simulations performed using moments up to the fourth order, while in Figure 5 the results illustrate simulations performed using moments up to the sixth order, and in Figure 6 the results are for simulations performed using moments up to the eighth order.

Comparing the results depicted in Figures 4-6, it is clear that, as expected, when increasing the number of moments, the oscillations of the particle velocity distribution function reconstruction decrease [1]. Furthermore, considering Figure 6, the numerical solution (obtained with moments up to the eighth order) shows a nice agreement with the analytical solution, although some oscillations are still present.

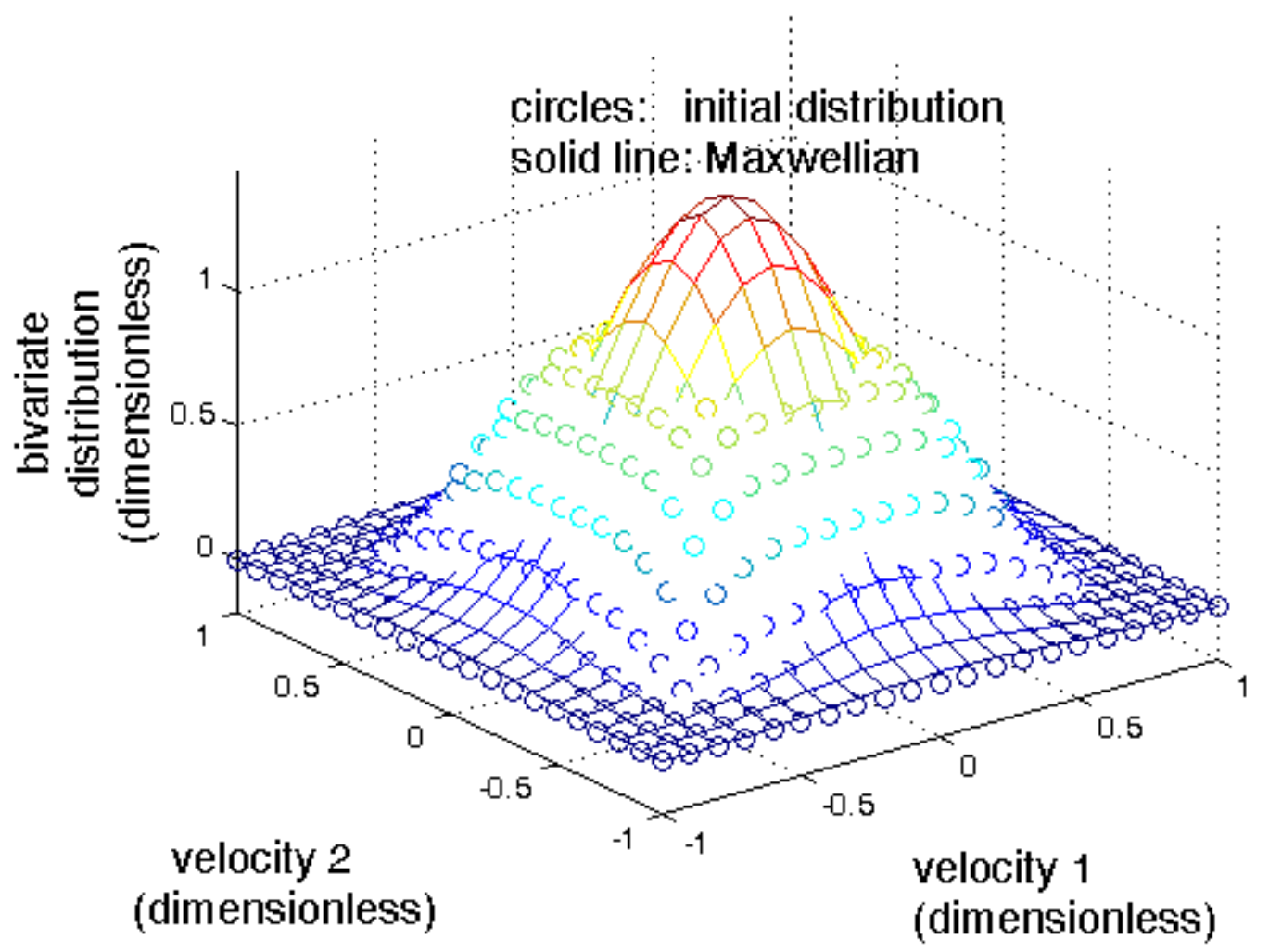

Figure 3 


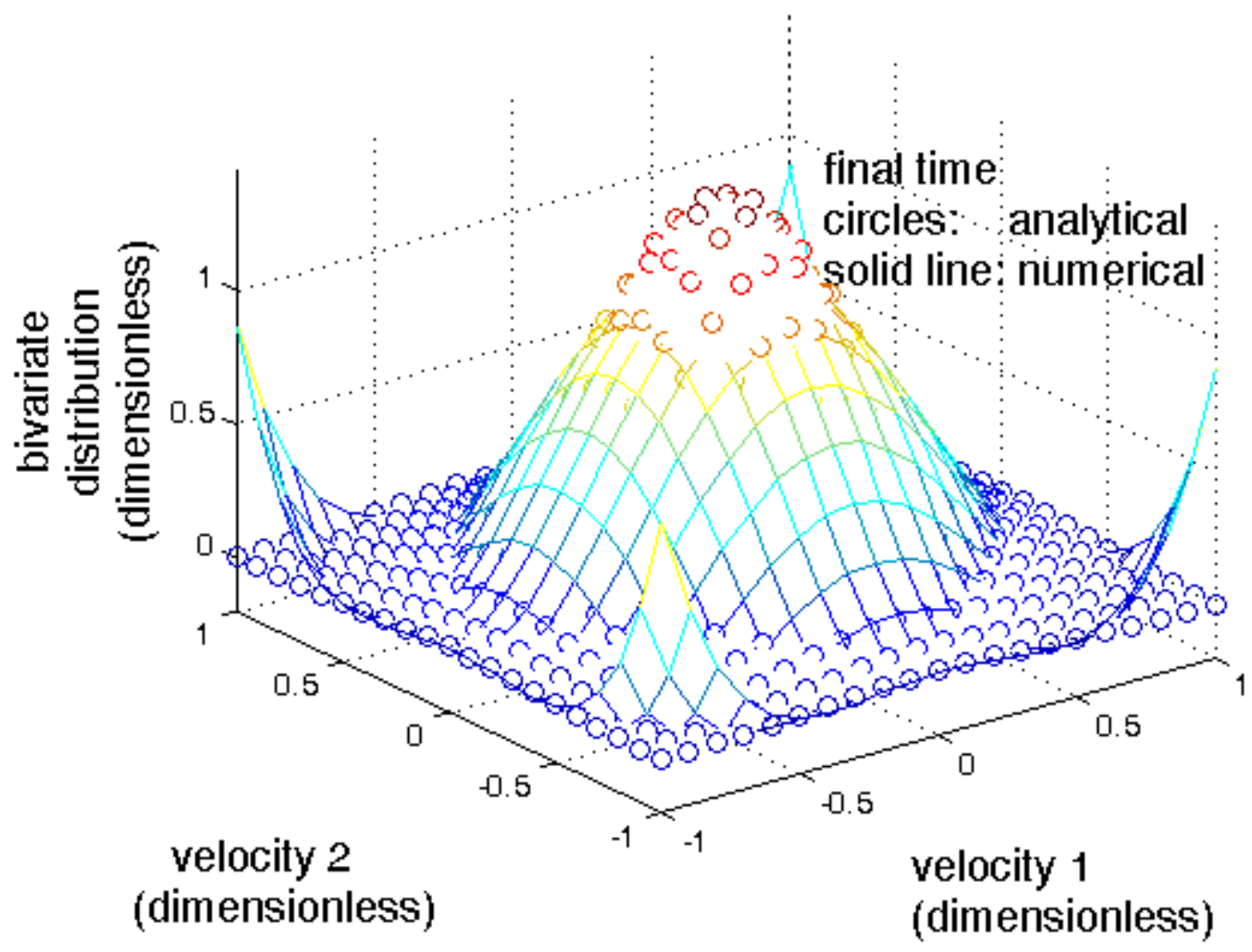

Figure 4 


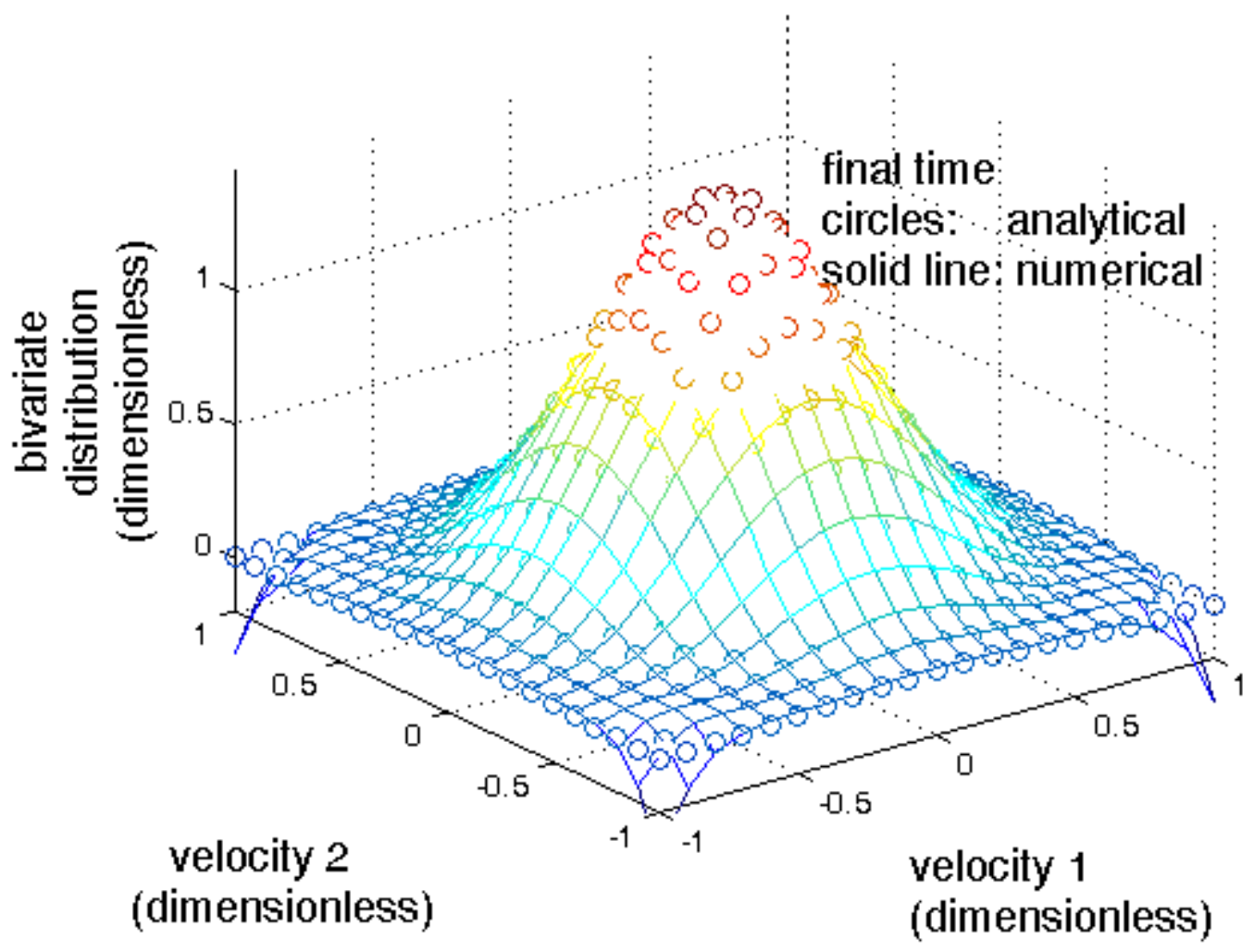

Figure 5 


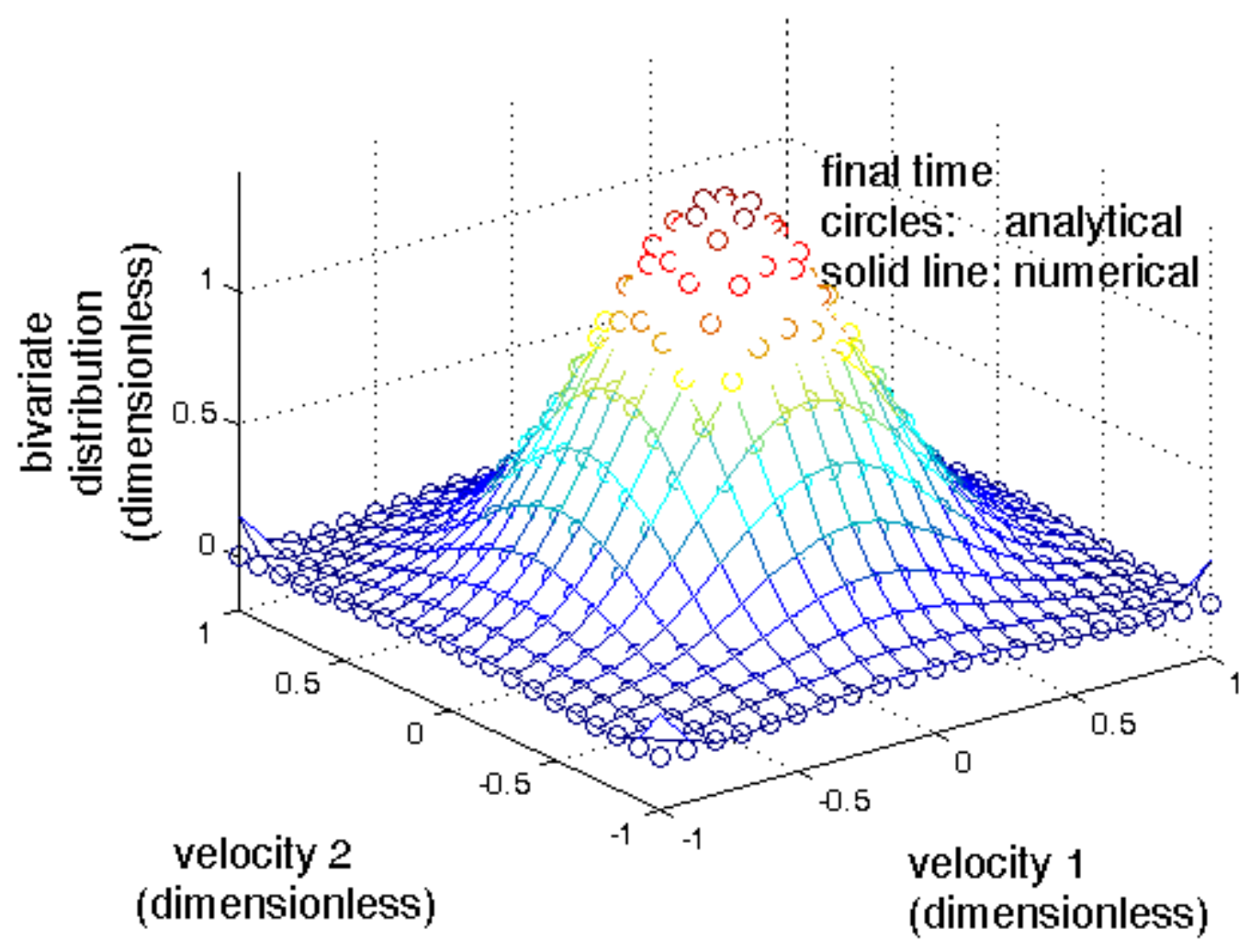

Figure 6

\section{FCMOM validation for the Boltzmann equation for in-homogeneous conditions}

The bi-dimensional Boltzmann equation (in in-homogeneous conditions) is the following equation:

$\frac{\partial f\left(\mathbf{c}_{1}, \mathbf{x}, t\right)}{\partial t}+\frac{\partial \mathbf{c}_{1} \cdot f\left(\mathbf{c}_{1}, \mathbf{x}, t\right)}{\partial \mathbf{x}}+\frac{\partial \mathbf{F} \cdot f\left(\mathbf{c}_{1}, \mathbf{x}, t\right)}{\partial \mathbf{c}_{1}}=\frac{\partial_{e} f}{\partial t}$

In Eq. (4), $t$ is the time, $\mathbf{x}$ is the (location) spatial coordinate, $\mathbf{c}_{\mathbf{1}}$ are the particle velocities, $f$ is the particle velocity distribution function, $\mathbf{F}$ are the total external forces (per unit mass) and $\frac{\partial_{e} f}{\partial t}$ is the collision integral (variation due to the particle encounters).

The focus here is on the solution of the collision-less Boltzmann equation (that is, $\frac{\partial_{e} f}{\partial t}$ is neglected), because the effect of the collision operator was investigated directly when assuming 
homogeneous conditions. Collision-less flow can be assumed in the dilute limit. Additionally, external forces are neglected; in this way the attention is directed towards the convective term [second term on the left-hand side of Eq. (4)], which is the critical term (numerically) of the collision-less Boltzmann equation.

Therefore, the following equation was considered:

$\frac{\partial f\left(\mathbf{c}_{1}, \mathbf{x}, t\right)}{\partial t}+\frac{\partial \mathbf{c}_{1} \cdot f\left(\mathbf{c}_{1}, \mathbf{x}, t\right)}{\partial \mathbf{x}}=0$

More specifically, a discussion of the application of the FCMOM to solve the Boltzmann equation in supersonic dilute conditions provided (since the particle phase in gas-solid flows is often in supersonic conditions).

When the moments-transformation was applied to Eq. (5) in 1-D ( $x$ coordinate), the dimensionless moments-evolution equations become:

$$
\frac{\partial \mu_{i}(x, t)}{\partial t}+\frac{c_{1, x, \max }-c_{1, x, \min }}{2} \cdot \frac{\partial \mu_{i+1}(x, t)}{\partial x}=0
$$

where $c_{1, x, \max }, c_{1, x, \min }$ are the maximum and minimum constant values of the particle velocity (in $x$ direction). The results shown in Figures $7 \& 8$ illustrate an application of the implementation of Eq. (6). A granular system, in supersonic conditions, was considered in a 1-D domain. At the beginning, in the whole domain the system was defined by an initial particle velocity distribution function (as shown in Figure 7). Then, a perturbation was applied. Figure 8 shows the comparison of the final exact solution and the numerical solution by the FCMOM, and the results show that an excellent agreement was obtained. This demonstrated that the FCMOM can be used to simulate particle flows in supersonic regimes. 


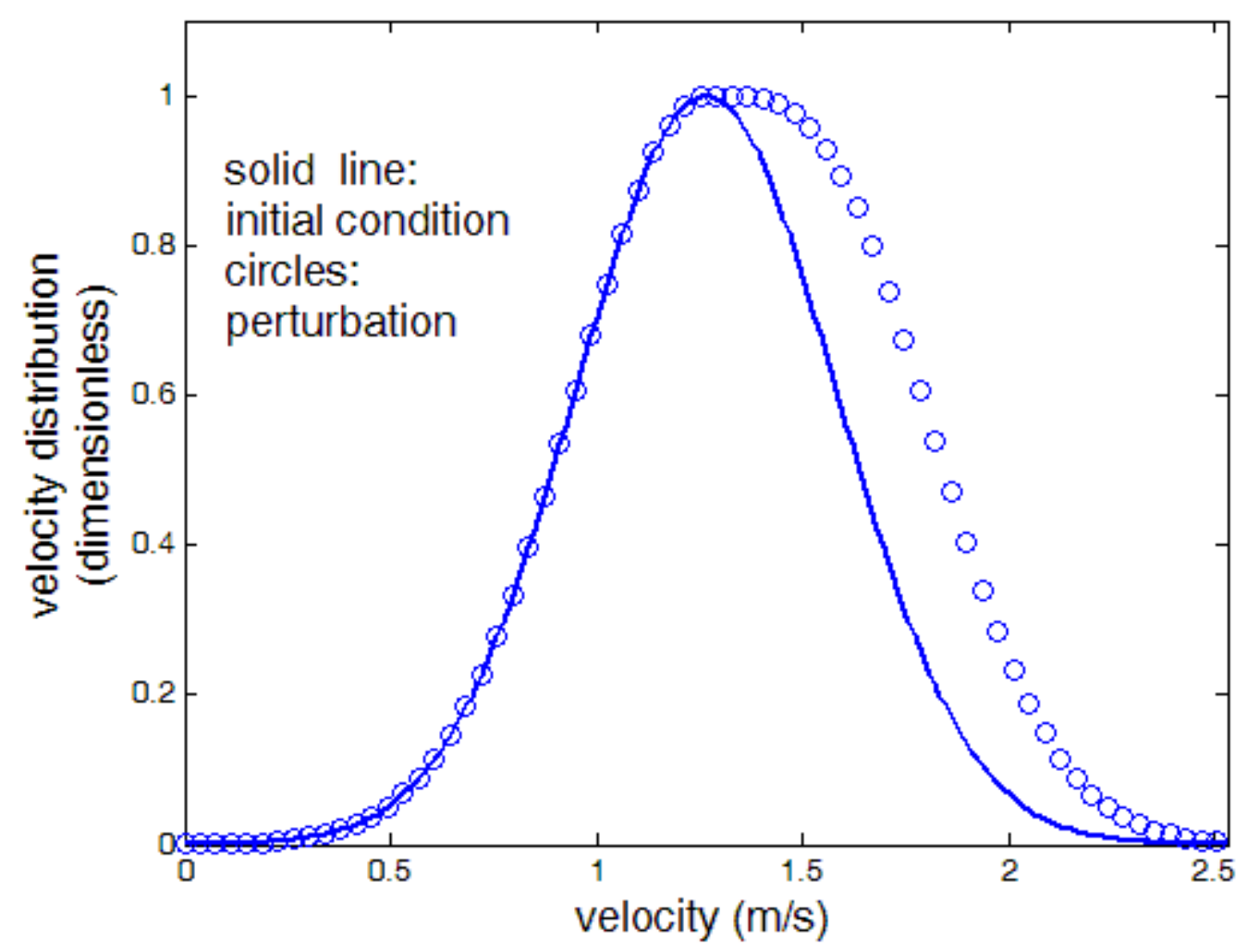

Figure 7 


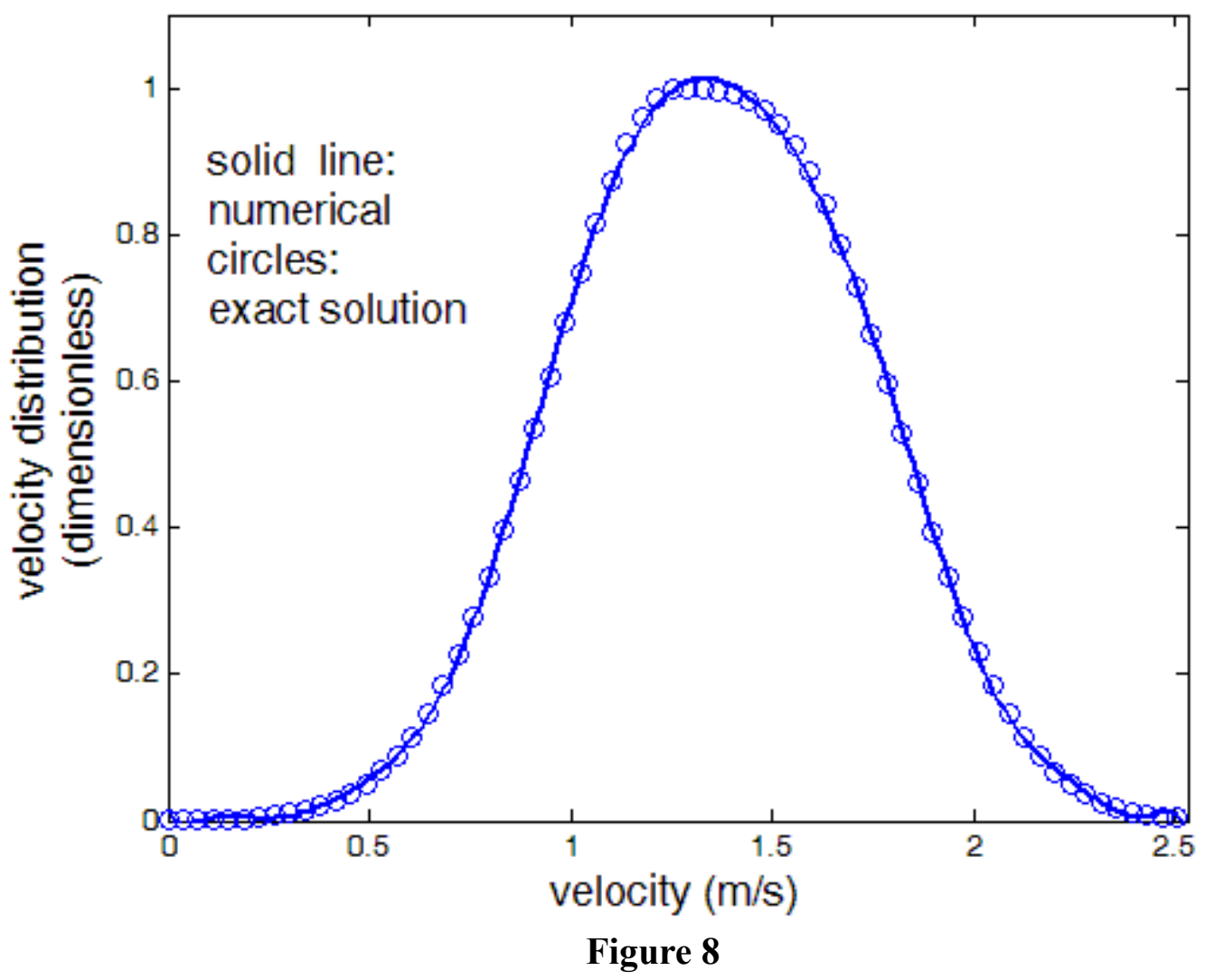

As another validation test for in-homogeneous conditions, the 2-D start up problem in dilute conditions was considered [5]. Specifically, the high Knudsen number limit was predicted by the solution of the collision-less Boltzmann equation. The low Knudsen number limit was predicted by the solution of the collision-less Boltzmann equation in the early-time behaviour and by a second-order slip model in the late-time behaviour.

Simulations were conducted regarding the start-up problem in dilute conditions (high Knudsen number). In the start-up problem, the system of particles was confined between two infinite walls. At time zero the walls start moving with a given velocity. In Figures $9 \& 10$, preliminary results are shown under the assumption 100 micron particle size and granular temperature of 0.1 $\mathrm{m}^{2} / \mathrm{s}^{2}$.

The results in Figure 9 illustrate the initial and final particle velocity distribution functions while Figure 10 shows the comparison between the numerical results and the exact solution for final time. 


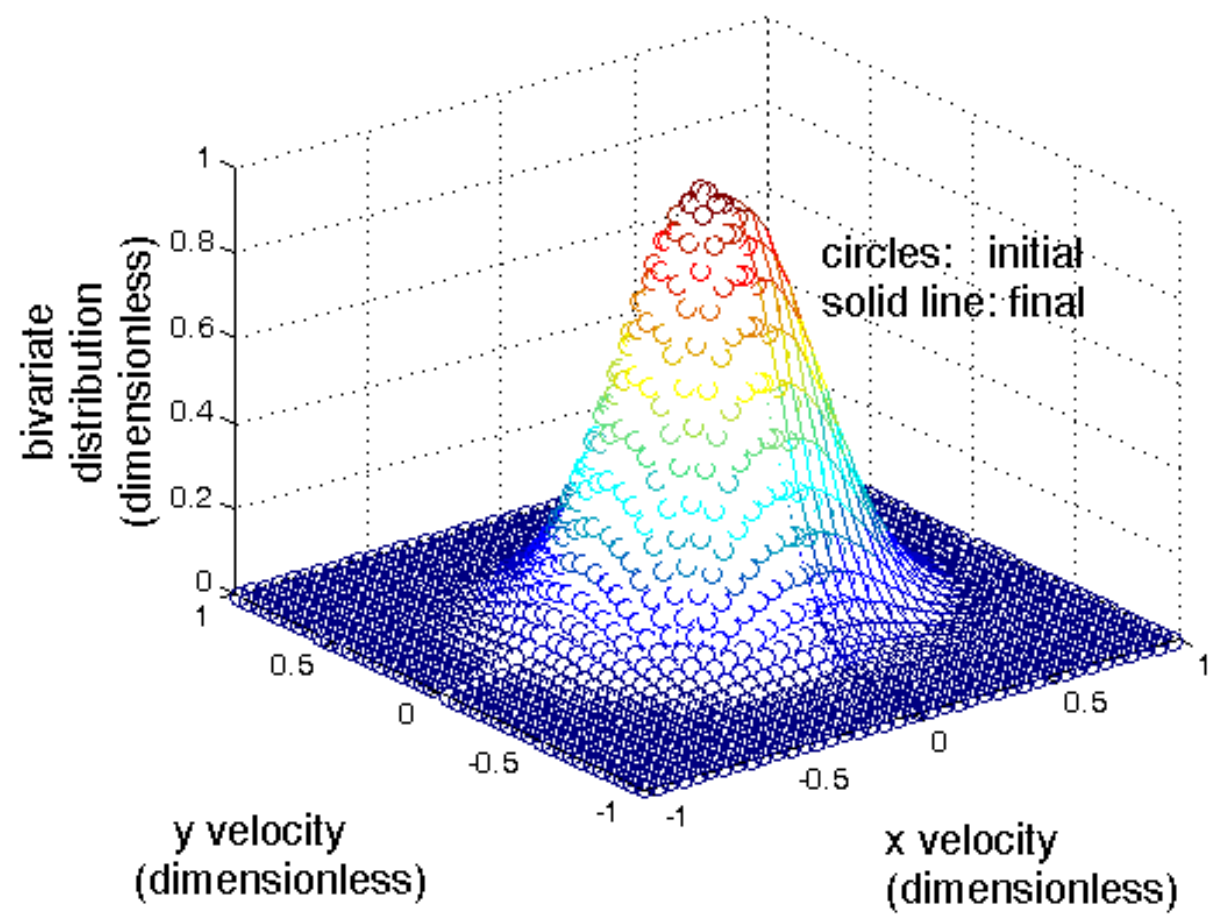

Figure 9

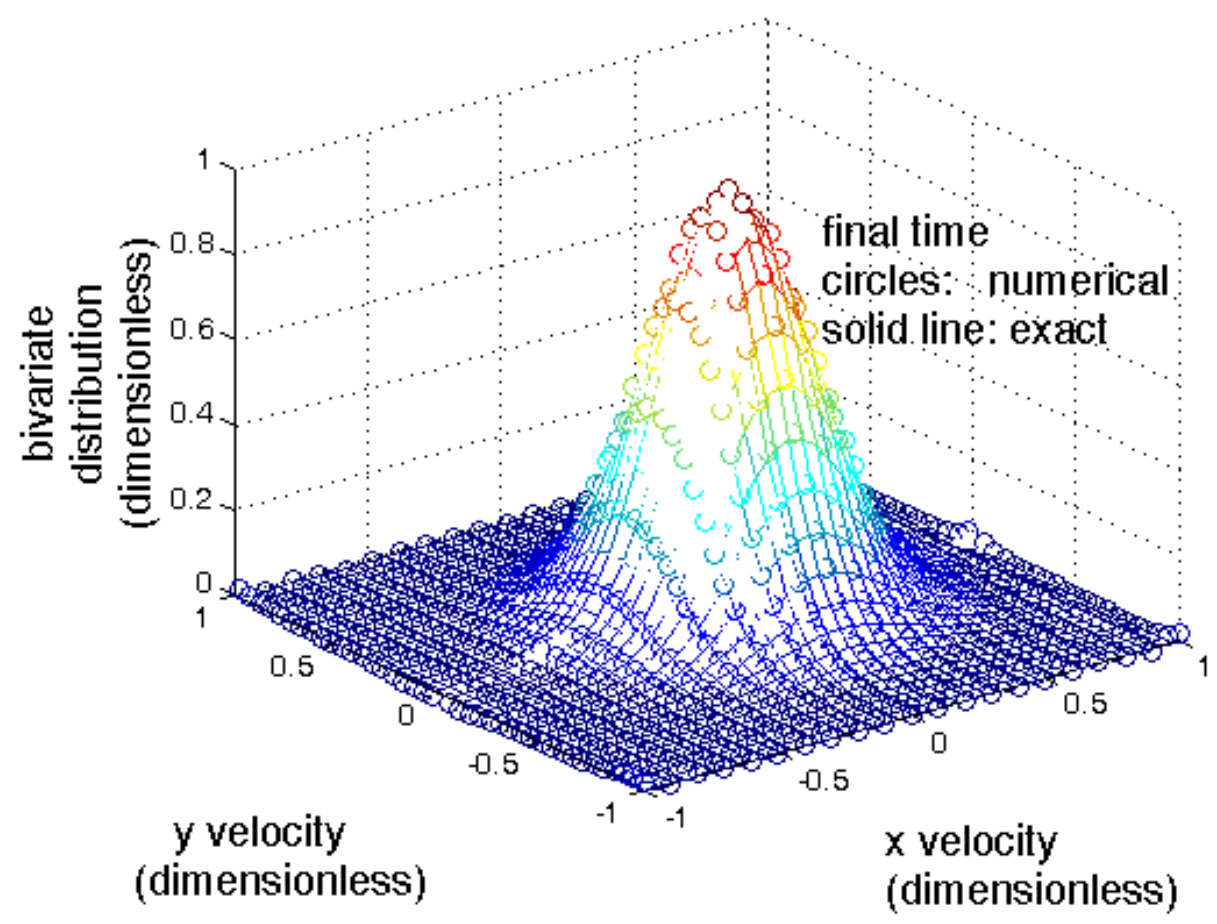

Figure 10 


\section{Transition between different regimes}

As already mentioned in the previous sections, several regimes need to be considered when validating a methodology to solve the Boltzmann equation. Neglecting the influence of the gas phase, two key parameters to identify the flow regimes are the Knudsen number and the Mach number. Low Knudsen numbers characterize relatively dense systems in which the collisions are dominant. Instead, high Knudsen numbers characterize rarified collision-less systems. The Mach number determines whether the flow is subsonic or supersonic. In granular flows and in fluidized beds, the flow can frequently be supersonic. While methodologies are available to solve correctly each of the regimes separately, it is difficult to capture and simulate correctly the transition between different regimes. It is therefore interesting to investigate the transition between different regimes, and how the available fluid dynamics models fail to simulate correctly such transition.

The classical continuum fluid dynamical approach is defined by the Navier-Stokes equations and it fails at high Knudsen numbers (roughly for Knudsen numbers above 0.01) and at high Mach numbers. This is demonstrated in the experimental work of Alsmeyer [6]. Argon can be considered as a system of small elastic particles in which the rotational energy can be neglected. Navier-Stokes simulations of one-dimensional shock waves cannot predict correctly the argon density profiles across the shock when increasing the Mach number. The maximum of the reciprocal shock thickness in argon (as a function of the Mach number) is between 0.25 and 0.3 , instead the Navier-Stokes simulations over-predict such quantity. Additionally, the NavierStokes approach fails in predicting the asymmetry of the density profiles, as a function of the Mach number. Finally, the flow anisotropic structure cannot be reproduced at the Navier-Stokes level. Boyd et al. [7] performed numerical simulations of one-dimensional normal shock waves (for systems in which the rotational energy is relevant). In such simulations, it is shown again that the continuum approach fails to predict correctly the shock thickness when increasing the Mach number. When the Mach number increases, the shock thickness first decreases, then it reaches a minimum and finally it increases. Correspondingly, the reciprocal of the shock thickness, as a function of the Mach number, has a maximum. In the simulations performed by such maximum is about $0.32 / 0.34$. 


\section{Riser Hydrodynamics: Anisotropy and Particle Kinetics}

Two-Fluid Models: Continuity-Force-Momentum and Energy Balances in Riser-section

The basic two-fluid formulation for gas-particles flow in risers is represented in the following balance equations [8].

$$
\begin{aligned}
& \frac{\partial}{\partial t}\left(\varepsilon_{g} \rho_{g}\right)+\nabla \bullet\left(\varepsilon_{g} \rho_{g} \mathbf{v}_{g}\right)-E_{i m}^{s}-S_{i m}^{s}=0 \\
& \frac{\partial}{\partial t}\left(\varepsilon_{s} \rho_{s}\right)+\nabla \bullet\left(\varepsilon_{s} \rho_{s} \mathbf{v}_{s}\right)+E_{i m}^{s}+S_{i m}^{s}=0 \\
& \frac{\partial}{\partial t}\left(\varepsilon_{g} \rho_{g} \mathbf{v}_{g}\right)+\nabla \bullet\left(\varepsilon_{g} \rho_{g} \mathbf{v}_{g} \mathbf{v}_{g}\right)=-\varepsilon_{g} \nabla P_{g}+\nabla \bullet \boldsymbol{\tau}_{g}-\beta\left(\mathbf{v}_{g}-\mathbf{v}_{s}\right)+\varepsilon_{g} \rho_{g} \mathbf{g}+E_{i M}^{s}+S_{i M}^{s} \\
& \frac{\partial}{\partial t}\left(\varepsilon_{s} \rho_{s} \mathbf{v}_{s}\right)+\nabla \bullet\left(\varepsilon_{s} \rho_{s} \mathbf{v}_{s} \mathbf{v}_{s}\right)=-\varepsilon_{s} \nabla P_{g}+\nabla \bullet \boldsymbol{\tau}_{s}+\beta\left(\mathbf{v}_{g}-\mathbf{v}_{s}\right)+\varepsilon_{s}\left(\rho_{s}-\rho_{g}\right) \mathbf{g}-E_{i M}^{s}-S_{i M}^{s} \\
& \frac{3}{2}\left[\frac{\partial}{\partial t}\left(\varepsilon_{s} \rho_{s} \theta\right)+\nabla \bullet\left(\varepsilon_{s} \rho_{s} \theta \mathbf{v}_{s}\right)\right]=\boldsymbol{\tau}_{s}: \nabla \mathbf{v}_{s}+\nabla \bullet\left(\kappa_{s} \nabla \theta\right)-\gamma_{s}
\end{aligned}
$$

Accompanying those balances are constitutive equations and material functions representing upto date developments [9].

$$
\begin{aligned}
& \boldsymbol{\tau}_{g}=2 \varepsilon_{g} \mu_{g}\left\{\frac{1}{2}\left[\nabla \mathbf{v}_{g}+\left(\nabla \mathbf{v}_{g}\right)^{T}\right]-\frac{1}{3}\left(\nabla \bullet \mathbf{v}_{g}\right) \mathbf{I}\right\} \\
& \boldsymbol{\tau}_{S}=\left(-P_{S}+\xi_{S} \nabla \bullet \mathbf{v}_{S}\right) \mathbf{I}+2 \mu_{S}\left\{\frac{1}{2}\left[\nabla \mathbf{v}_{S}+\left(\nabla \mathbf{v}_{S}\right)^{T}\right]-\frac{1}{3}\left(\nabla \bullet \mathbf{v}_{S}\right) \mathbf{I}\right\} \\
& P_{S}=\varepsilon_{S} \rho_{S} \theta\left[1+2(1+e) g_{O} \varepsilon_{S}\right] \\
& \xi_{S}=\frac{4}{3} \varepsilon_{S}^{2} \rho_{S} d_{p}(1+e) g_{o} \sqrt{\frac{\theta}{\pi}} \\
& \mu_{S}=\frac{10 \sqrt{\pi} \rho_{S} d_{p} \sqrt{\theta}}{96(1+e) g_{o}}\left[1+\frac{4}{5}(1+e) g_{0} \varepsilon_{S}\right]^{2}+\frac{4}{5} \varepsilon_{S}^{2} \rho_{S} d_{p}(1+e) g_{o} \sqrt{\frac{\theta}{\pi}} \\
& \kappa_{S}=\frac{150 \sqrt{\pi} \rho_{S} d_{p} \sqrt{\theta}}{384(1+e) g_{o}}\left[1+\frac{6}{5}(1+e) g_{0} \varepsilon_{S}\right]^{2}+2 \varepsilon_{S}^{2} \rho_{S} d_{p}(1+e) g_{O} \sqrt{\frac{\theta}{\pi}} \\
& \gamma_{s}=3\left(1-e^{2}\right) \varepsilon_{s}^{2} \rho_{s} g_{o} \theta\left(\frac{4}{d_{p}} \sqrt{\frac{\theta}{\pi}}-\nabla \bullet v_{S}\right) \\
& \beta=\frac{3}{4} C_{d} \frac{\varepsilon_{s} \rho_{g}\left|\mathbf{v}_{g}-\mathbf{v}_{s}\right|}{d_{p}} \varepsilon_{g}^{-2.65} \rightarrow \varepsilon_{g} \geq 0.8 \\
& C_{d}=\frac{24}{\operatorname{Re}_{s}}\left(1+0.15 \mathrm{Re}_{s}^{0.687}\right) \rightarrow \operatorname{Re}_{s}<1000 \\
& C_{d}=0.44 \rightarrow \operatorname{Re} \geq 1000 \\
& \beta=150 \frac{\varepsilon_{s}^{2} \mu_{g}}{\varepsilon_{g}^{2} d_{p}^{2}}+1.75 \frac{\rho_{g} \varepsilon_{s}\left|\mathbf{v}_{g}-\mathbf{v}_{s}\right|}{d_{p} \varepsilon_{g}} \rightarrow \varepsilon_{g}<0.8 \\
& E_{i m}^{s}, E_{i M}^{s}, S_{i m}^{s}, S_{i M}^{s}(?)
\end{aligned}
$$


Numerous forms of (the above) hydrodynamic model have been tested by expert research groups under variant circumstances ([10-12] among others). The balance equations provided include excess terms for sake of completeness in order to account for reaction and/or mass transfer between phases (as observed in coal gasification). The excess terms can be checked for consistency with a region or mixture balance since they must disappear when two balances (for fluid and solid phases) are added. The region or mixture balance provides a practical tool to check consistency of the individual balances and to make sure that the excess terms are eliminated (when balances are added). The balance for energy dissipation is in fact a region balance. Together with thermodynamic restrictions on the balances and their constitutive equations the region balance is a valuable tool for analysis when experimental data predicts region rather than individual phases. And it provides a restriction on the macroscopic balances when mezzoscale balances (two-fluid model equations) are averaged over the global volume. A lot of reported works in the open literature in which similar formulations are presented either disregard or fail this consistency check on the balances. It thus becomes a difficult task to generalize results of data in those articles. However, at some point all accumulated research effort will have to converge at some point.

Adapting two-fluid (TF) models to specific cases: reflections and challenges: It is noted that the basic balance equations in TF models are pretty universal and fairly rigorous to describe multiphase flow behavior. The results of case studies of hydrodynamics of risers have shown that it is still difficult to define riser flow regimes (contrary to what is known about fluid flow regimes, ranging from laminar to turbulent flow). The major challenge then is how to adapt TF models to specific gas-particles flow problems, especially given the fact that such flows range from stationary fluidized state, dense (pneumatic) granular flows, riser flows and onto (dilute particle) entrainment flows. Unfortunately, many excellent reported works do not offer any distinction between fluidized state and riser flows. In this work gas-particles flows in risers is considered to fall between fast fluidized state and entrainment. Another issue to consider is particles concentration and definition of crossover regions between dilute to dense flows where particles collisions are significant or some particles flow as clusters. The remaining challenge is related to description of material functions (constitutive equations) and specifically how to incorporate particle kinetics, experimental and theoretical verification of the assumptions used to develop the models, and scale-up issues. Pending answers to these challenges we are still far off from accurate prediction with duplicable results.

Even though analysis of momentum re-distribution in risers is still ongoing, we will make some observations (or postulates) about riser flows:

1) Gas phase flow is continuous and is accurately predictable in risers through tractable data like pressure drop, fluid and mass velocities, friction factor and etc. What this means also is that we can impose the no-slip boundary condition at the wall.

2) Particles phase on the other hand is not continuous. This means analysis must take into account discrete particle-particle collisions, particles collisions with the wall and collisions between clusters of particles. Furthermore, the no-slip boundary condition cannot be imposed on particles phase in simple terms, other considerations such as flux of granular temperature at the wall being equated to dissipation at the wall have to be taken into account [13]. 
In relation to particles phase there are few tractable in-situ data that can track particle kinetics even though solids mass velocity, porosity, particles imaging and a few other data can be extracted in addition to batch rheological properties. Due to the inherent nature of the two phases in a riser we still need to find answers to:

- How to model solid/particles phase stresses for non-rigid and non-stationary particles phase?

- How model the drag force term given the fact that particles are pneumatically transported by the fluid fronts? Or in other words, what is the order of magnitude of the relative velocity in relation to say gas velocity?

These questions were easily answered in the large volume of works accumulated so far on stable fluidized beds based upon the two-fluid model approaches. However, for riser flows and dense multiphase flows one needs to exercise caution on extending data extracted based on assumption of fluidized state. The conclusions on momentum re-distribution in gas-particles flow in a riser are supported by a wealth of data in the literature. The studies show that when particles collide with the wall surfaces the particles dissipate some of the energy and lose momentum and since particles are not a continuous phase the collisions with other particles migrating from the center become more often (since particles are pneumatically transported by the fluid losing momentum at the wall) and some particles then have a tendency of bouncing off others in somewhat downward spiral relative to the upward fluid flow. Particle-particle and particle-wall collisions do henceforth contribute to observed segregation in risers in addition to momentum loss at the wall by the gas. The bouncing off the wall and particle-particle collisions indicates also that relative velocity is higher closer to the wall surface and subsequently the drag force close to the wall will also increase in comparison to that at the center of the riser. Similar to stable fluidized beds, there is also excess gas (above what is necessary to support pneumatic transport of particles phase) together with above-mentioned particles collisions can lead to segregation in risers [14].

Even with those restrictions or assumptions about the two phases in riser flows, the two-fluid model coupled with kinetics theory simulations has provided insight into localized transport phenomena (at continuum scale) and particle kinetics level. However, one additional constraint that must be placed on the two-fluid models is that averaged over the area and global volume the balances should collapse into macroscopic scale balances tractable through experimentally accessible data. It is for this reason that sometimes mechanistic arguments and analysis can be made to study riser hydrodynamics in the cause of advancing knowledge (even though the approach is ad hoc).

Multiphase Flow with Unequal Granular Temperatures: Through simulation and experimental studies the Gidaspow group contributed to this work by studying multiphase flow with unequal granular temperature and particle sizes and masses. This study advanced knowledge on anisotropy or particles kinetics and was based on extending concepts in multiphase kinetic theory approach especially development of expressions for number of binary collisions, viscosities and conductivities. It provided insights into particle collisions in which normal and tangential restitution coefficients are considered. The attached article (provided as an Appendix to this report) includes the major findings on granular temperature, and solid volume fraction [15]. 
Incremental Force-Momentum balance in a riser: One of the challenges and difficulty of implementing two-fluid models has to deal with verifying the assumptions about particle-particle and fluid-particle interactions. In this work force- momentum balances were presented in order to aid in interpretation of macroscopic data for validation cases in the literature. The balances were derived via mechanistic analysis of force-momentum balance in a riser. This approach was more simple and intuitive to use than simply taking area average of the two-fluid balances [in which case it would require significant assumptions]. This approach also brings us closer to the limiting case of analysis of fluid-like flow in pipes when the system is significantly less dense particle-wise [16].

$$
\begin{aligned}
& -\frac{1}{r} \frac{d}{d r}\left(r \tau_{r z}^{g}\right)+\frac{\varepsilon_{g}\left(P_{0}-P_{L}\right)-\varepsilon_{g} \rho_{g} L g}{L}-\beta\left(v_{z}^{g}-v_{z}^{s}\right)=0 \\
& -\frac{1}{r} \frac{d}{d r}\left(r \tau_{r z}^{s}\right)+\frac{\varepsilon_{s}\left(P_{0}-P_{L}\right)-\varepsilon_{s} \rho_{s} L g}{L}+\beta\left(v_{z}^{g}-v_{z}^{s}\right)=0
\end{aligned}
$$

The above equations are for the gas and solid phase momentum balances. Note that additional terms may be needed for dense systems to account for particle pressure and collision terms. But in a macroscopic sense those terms are yet to be quantified or defined. Besides, these equations are an over-simplification as far as evidence of observed particle kinetics. But then again, at macroscopic scale it is difficult to quantify some of the terms in explicit sense. These equations are subject to boundary conditions in the core and annular regions of the riser:

- $\quad$ BC1: at $r=0, \quad r \tau_{r z}^{g}=0 \& r \tau_{r z}^{s}=0$

- BC2: at $r=R, \quad v_{z}^{g}=0$ (no-slip condition) \& use BCs from [13]

The second boundary condition for particles is generated from information about flux of granular temperature and particle energy dissipation at the wall [13]. Some validation cases in the literature support the no-slip condition assumed for the gas phase [17] while some cases provide insights into validity of momentum-transfer coefficient [18]. However, there is still analysis to be made regarding drag force term and how it should be accounted for in momentum redistribution in gas-particles flow in a riser. The results of this analysis will be presented later [14]. A good discussion on the boundary conditions at the wall, for both limiting and (simplified) kinetic theory, was provided by Jackson [11].

Availability of data and validation cases that support segregation in risers is critical to development and improvement in multiphase theory approaches. In general any data on internal profiles of measured and predicted radial profiles (voidage, velocities, etc.) should provide more insights into accurate interpretation of momentum re-distribution due to solid pressure and viscosity (or particles rheology), momentum transfer coefficient, especially their interpretation in macroscopic sense. As previously stated (in prior sections of this report) the region balances serve as a valuable tool for interpreting literature data. From such analysis it was readily observed that the net pressure force must equal the sum of the viscous forces and drag force. When the two balance equations (for gas \& solids) are summed up we obtain a region or mixture momentum balance 


$$
-\frac{1}{r} \frac{d}{d r}\left(r \tau_{r z}^{g}+r \tau_{r z}^{s}\right)+\frac{\left(P_{0}-P_{L}\right)-\left(\varepsilon_{g} \rho_{g}+\varepsilon_{s} \rho_{s}\right) L g}{L}=0
$$

where

$$
\frac{1}{r} \frac{d}{d r}\left(r \tau_{r z}^{g}+r \tau_{r z}^{s}\right) \text { is the net rate of momentum transfer per unit volume. }
$$

When Eq. (9) is integrated over the cross-section area we observe that

$$
\left.\left(r \tau_{r z}^{g}+r \tau_{r z}^{s}\right)\right|_{0} ^{R}=\left[\frac{\left(P_{0}-P_{L}\right)-\left(\varepsilon_{g} \rho_{g}+\varepsilon_{s} \rho_{s}\right) L g}{L}\right] R^{2}
$$

Equation (10) essentially indicates that there is momentum loss and it is equal to the net pressure drop on the riser (after subtracting the buoyant forces). It also shows that the combined shear stress, as a function of radius, is linear from the core to the annular region. It should be noted that these equations over-simplify the complicated gas-particle and particle-particle interactions observed in several studies. But they are used as baseline interpretation of macroscopic data. It should be noted that the terms on the right hand side of Eq. (10) are measurable entities and fluid shear stress can be predicted from correlation of $f_{g}$, the friction factor. These terms are obtained from cold-flow model simulations or experimental data and can give insights into the particle shear force at the boundary [14].

\section{Two correlating and scaling parameters:}

- Reynolds number: $\operatorname{Re}_{s}=\frac{\varepsilon_{g} \rho_{s}\left|v_{z}^{g}-v_{z}^{s}\right|}{\mu_{g}}$

- Superficial velocity is defined from either volumetric flow or mass velocities

o $\quad \dot{V}_{T}=\pi R^{2}\left(\frac{\bar{v}_{z}^{g}}{\varepsilon_{g}}+\frac{\bar{v}_{z}^{s}}{\varepsilon_{s}}\right), \bar{v}_{z}$ is superficial velocity \& $\dot{V}_{T}$ is total volumetric rate. Here $\bar{v}_{z}^{g}=\bar{W}_{g} /\left(\varepsilon_{g} \bar{\rho}_{g}\right) \& \bar{v}_{z}^{s}=\bar{W}_{s} /\left(\varepsilon_{s} \bar{\rho}_{s}\right)$

o $\dot{M}_{T}=\pi R^{2}\left(G_{g}+G_{s}\right) \equiv \pi R^{2}\left(W_{g}+W_{s}\right)$ where $G \& W$ are mass velocities \& fluxes, respectively.

In general the superficial velocities are correlated to the average velocities if the velocity profiles are available:

$$
\bar{v}_{z}^{g}=\frac{\int_{0}^{R} v_{z}^{g}(r) 2 \pi r d r}{\varepsilon_{g} \int_{0}^{R} 2 \pi r d r} \& \bar{v}_{z}^{g}=\frac{\int_{0}^{R} v_{z}^{s}(r) 2 \pi r d r}{\varepsilon_{s} \int_{0}^{R} 2 \pi r d r}
$$

Note that in this case we need expressions of velocities at every radial position.

Velocity profiles: On the other hand, simulation studies based on two-fluid models coupled with kinetic theory constitutive expressions have to a certain extent been able to provide insights 
velocity distribution in risers. However, mechanistic arguments can help reveal the limitations of two-fluid models especially when viewed from the point of view of verifiable macroscopic data. Velocity profiles under limiting conditions are obtained from solving the following equations

$$
\begin{aligned}
& \frac{1}{r} \frac{d}{d r}\left(r \mu_{g} \frac{d v_{z}^{g}}{d r}\right)+\frac{\varepsilon_{g}\left(P_{0}-P_{L}\right)-\varepsilon_{g} \rho_{g} L g}{L}-\beta\left(v_{z}^{g}-v_{z}^{s}\right)=0 \\
& \frac{1}{r} \frac{d}{d r}\left(r \mu_{s} \frac{d v_{z}^{s}}{d r}\right)+\frac{\varepsilon_{s}\left(P_{0}-P_{L}\right)-\varepsilon_{s} \rho_{s} L g}{L}+\beta\left(v_{z}^{g}-v_{z}^{s}\right)=0
\end{aligned}
$$

Or from the mixture momentum balance, Eq. (9), under limiting conditions: in the core or annular regions.

It was shown from validation case that Poiseuille type solution is readily obtained by considering limiting solution (case) of Eq. (9). For example, in a thin annular region one obtains the solid velocity profile

$$
v_{z}^{s}=2 \bar{v}_{z}^{s}\left(1-\frac{r^{2}}{R^{2}}\right)
$$

where

$$
\bar{v}_{z}^{s}=\frac{W_{s}}{\varepsilon_{g} \rho_{s}}
$$

The solution was successfully validated for this model with experiments [19]. Some insights into the relative sizes of the annular and core regions will be published later [14]. In these studies we are also looking at using a Hookian model for the solids stress term. In this model we assume that solid stress is modeled from some form of strain represented by porosity differences (leading to bed expansion or segregation as such). Why such a model? The reason is because the particulate phase is non-rigid and in this case sometimes behaves as discrete particles.

Now some insights into obtaining velocity profiles via solution of Eq. (11a) - utilizing a model of relative velocity - It is clear that we would need to solve simultaneously Eqs. (11a) \& (11b). But this would present some difficulties if we wish to obtain a closed-form (analytical) solution (even under limiting cases). The equations are coupled through the drag-force term. It is should be clear why the mixture balance or region mixture balance equation is simpler to handle under limiting case [19]. The model for drag force and for that matter relative velocity has been well studied both experimentally and theoretically. It is used in predictions in filtration, porous media and fluidized bed transport. In dilute pneumatic transport and riser flows particularly we should note that particles more less move with the fluid. Thus, unless there are many particle collisions (at the same time scale gas flow), it can be assumed that the ratio of velocities from the core to the annular region is constant. And that the ratio is constant. This implies that Eqs. (11a) \& (11b) can be de-coupled under limiting conditions. Thus the equations would become 


$$
\begin{aligned}
& \frac{1}{r} \frac{d}{d r}\left(r \mu_{g} \frac{d v_{z}^{g}}{d r}\right)+\frac{\varepsilon_{g}\left(P_{0}-P_{L}\right)-\varepsilon_{g} \rho_{g} L g}{L}-\beta_{g} v_{z}^{g}=0 \\
& \frac{1}{r} \frac{d}{d r}\left(r \mu_{s} \frac{d v_{z}^{s}}{d r}\right)+\frac{\varepsilon_{s}\left(P_{0}-P_{L}\right)-\varepsilon_{s} \rho_{s} L g}{L}+\beta_{s} v_{z}^{s}=0
\end{aligned}
$$

where $\beta_{g}=\beta(1-\psi)$ and $\beta_{s}=\beta\left(\frac{1}{\psi}-1\right)$ are assumed to be constant [this is just one of the assumptions yet to be supported by validation cases - so it is based on intuition for less dense system]. Pending those validation cases and data velocity profiles are computed using the rigorous approaches based on two-fluid model and kinetic theory. However, the mechanistic presentation allows us to provide insights into what is supported by data in a macroscopic sense.

The other case that was considered to solve for velocity profiles via simultaneous solution of Eqs. (11a)\& (11b). Methods are still being sought for solving the equations for specific limiting conditions (again for interpreting macroscopic data). But still one needs to analyze some quality experimental data on riser flows. In this regard the validity of Ergun-type and Wen \& Wu type correlations for momentum-transfer coefficient $(\beta)$ have to be re-examined in light of what we have concluded about the relevance of the drag force term. Drag force for deformable solids, non-deformable solids, dilute particle phase and dense particle phase should be differentiated. The interplay among buoyant forces, drag force and particle acceleration/deceleration as a result of momentum re-distribution should be critically examined.

\section{CONCLUSION}

The accurate prediction of gas-solid flows in risers will ultimately involve coordinated research efforts and convergence of ideas from different research groups. Certainly the Department of Energy through NETL research activities, particularly through the MFIX codes and data generation, is accelerating these efforts. In general, the ideas are to come from development of efficient numerical schemes (such as FCMOM), further developments in particles kinetic theory approaches and further understanding of verifiable assumptions to be imposed on two-fluid models.

The ultimate objective of the original proposed work was "to derive the governing and constitutive equations based on the kinetic theory for PSP by using a generalized Boltzmann equation (GBE) in which both particle size and velocity distributions is taken into account”. What was accomplished of this undertaking was to develop the FCMOM technique and validate it for several cases. In the meantime, some effort was expended to advance knowledge on riser hydrodynamics and specifically how to handle anisotropy or particles kinetics and its effect on segregation. The idea here is that since the basis for predicting riser flows is based on two-fluid model formulations coupled with (multiphase) kinetic theory there is a need to re-formulate the models in order to make them computationally manageable but at the same time the solutions have to be backed up by limiting macroscopic data. 
This work also demonstrated that multiphase kinetic theory can be used to simulate multiphase flows with unequal granular temperatures and unequal particle sizes and masses. This approach required new expressions for binary collisions, viscosities and conductivities in order to simulate two particle sizes with unequal mass and size and unequal granular temperatures (by assuming normal and tangential restitution coefficients). The theoretical assumptions were verified experimentally on the IIT riser.

Finally, the analysis of momentum re-distribution, owing to particle-particle and fluid-particle interactions in a riser, brought forth some limitations of two-fluid models especially the constitutive expressions for drag force coefficient and models of particulate phase stress. It should be noted that in riser flows there is no cumulative particulate phase stress especially considering that there is intense particle-particle collisions. The collisions lead to residual stresses due to particle-particle momentum exchanges. In general it is proposed that residual stresses and particle pressure can be modelled by a Hookian model or some generic stress-strain relation where the strain is expressed in some porosity difference between stressed and unstressed porosity. Thus, residual stress is a function of porosity. In general it is suggested that the drag force coefficient be determined from internal porosity and pressure-drop profiles while porosity and pressure profiles are needed to determine parameters in the residual stress term.

\section{REMAINING TASKS}

In the original Project Management Plan the FCMOM or generalized method of moments (GMOM) was to be implemented in MFIX for riser simulations. The remaining tasks are as follows.

\section{Incorporation of governing equations in MFIX}

Once the resulting governing partial differential equations are derived based on the kinetic theory for poly-dispersed systems they can then be incorporated into MFIX Code. Then the simulations should be compared with available data or computer simulation cases in the literature to test the validity of the governing equations.

\section{Simulation of Coal Conversion Processes}

The other remaining task is to simulation the flow behaviour of a poly-dispersed system in the riser section of a circulating fluidized bed. We hope that the generalized method of moments (GMOM) can simulate particle size distribution (PSD) evolution, when the particles change size or density due to heterogeneous reaction (e.g., coal conversion or gasification process).

\section{LITERATURE CITED}

[1] Montanero, J. M., Santos, A., “Computer simulation of uniformly heated granular fluids”, Granular Matter 2, 53-64, 2000.

[2] Strumendo, M., Arastoopour, H., "Solution of PBE by MOM in Finite Size Domains", Chem. Eng. Sci., 63(10), 2624, 2008. 
[3] Strumendo, M., Arastoopour, H., "Solution of Bivariate Population Balance Equations Using the Finite Size Domain Complete Set of Trial Functions Method of Moments (FCMOM)", Ind. Eng. Chem. Res., 48(1), 262-273, 2009.

[4] Ramkrishna, D., Population Balances: Theory and Applications to Particulate Systems in Engineering, Academic Press, London, 2000.

[5] Hadjiconstantinou N. G., Al-Mohssen H. A., "A linearized kinetic formulation including a second-order slip model for an impulsive start problem at arbitrary Knudsen numbers”, J. Fluid Mech., 533, 47-56, 2005.

[6] Alsmeyer H., "Density profiles in argon and nitrogen shock waves measured by the absorption of an electron beam”, J. Fluid Mech., 74, 497-513, 1976.

[7] Boyd I. D., Chen G., Candler G. V., "Predicting failure of the continuum fluid equations in transitional hypersonic flows", Physics of Fluids, 7, 210-219, 1995.

[8] Bogere M. N., "A rigorous description of gas-solid fluidized beds”, Chem. Eng. Sci., 51(4), 603, 1996.

[9] Tartan M., Gidaspow D., "Measurement of granular temperature and stresses in risers", AIChE J., 50, 1760-1775, 2004.

[10] Gidaspow D., Multiphase flow and fluidization. Continuum and kinetic theory descriptions, Academic Press, New York, 1994.

[11] Jackson R., The Dynamics of Fluidized Particles, Cambridge Univ. Press, Cambridge, UK, 2000.

[12] Yang Y., Arastoopour H., "Volume averaged governing equations for the fluid-solid Systems”, presented at the AIChE National Meeting, Chicago, 1990.

[13] Johnson, P. C., Jackson, R., "Frictional-collisional constitutive relations for granular materials, with application to plane shearing”, J. Fluid Mech., 176, 67, 1987.

[14] Bogere, M. N., etc., "Overlapping continua in gas-particles flows in a riser", under preparation.

[15] Songprawat, S., Gidaspow, D., "Multiphase flow with unequal granular temperatures", Chem. Eng. Sci., 65, 1134, 2010.

[16] Fahien, R. W., Fundamentals of Transport Phenomena, McGraw-Hill, New York, 1983.

[17] Martin, M. P., Turlier, P., Bernard, J. R., "Gas and solid behavior in cracking fluidized beds", Powder Technol. 70, 249, 1992.

[18] Hartge, E.-U., Ratschow, L., Wischnewski, R., Werther, J., "CFD-simulation of a circulating fluidized bed riser”, Particuology, 7, 283-296, 2009.

[19] Tartan, M. , Gidaspow, D., "Measurement of granular temperature and stresses in risers", AIChE J., 50, 1760-1775, 2004. 